\title{
Neue Kommunikationstechnologien und Wissenschaft: Veränderungspotentiale und Handlungsoptionen auf dem Weg zur Cyber-Wissenschaft
}

\section{Vom Füllen einer Forschungslücke ${ }^{1}$}

Wissenschafts- und Technikforschung („Science and Technology Studies" STS) ist ein vielgestaltiges und zugleich offenes Feld. Zumeist liegt der Fokus der STS-Studien entweder auf der Wissenschaft (wie kommen die Wissenschafter zu ihren Ergebnissen?) oder auf der Technik (wie reagiert die Gesellschaft auf eine neue Technologie oder wie wird die Technologieentwicklung durch ihr Eingebettetsein in der Gesellschaft beeinflusst?). Seltener geht es um beides gleichzeitig, also um Technologienutzung in der Wissenschaft. Die Beiträge der STS-Forschung zum Einsatz Internet-basierter Kommunikations- Forschungstechnologien in den Wissenschaften sind daher zumeist sehr spezifisch und betreffen nur einzelne Aspekte des Gesamtthemas, etwa die technologiegestützte Zusammenarbeit auf Distanz (z. B. Finholt/Brooks 1997; Walsh 1997; Olson et al. 2006), digitale Bibliotheken (z. B. Kilker/ Gay 1998), Fachinformationssysteme (z. B. Fröhlich 1993), die Rolle elektronischer Medien für die Fachkommunikation (Kling/McKim 1999; Fry 2003), die Rolle von neuen Forschungstechnologien (Beaulieu 2001; Joerges/Shinn 2001; siehe schon Bonitz 1979), E-mail-Listen (z. B. Matzat 2001) oder die Perspektiven für die Szientometrie (z. B. Harter 1996; Zelman 2002) und Indikatorenforschung (Leydesdorff/Scharnhorst 2003). Auch der jüngst erschienene Sammelband zu E-Science (Hine 2006) vereint vor allem Einzelbeiträge. Was bislang fehlte war eine umfassende Studie, die die vielen Lücken in der eher spärlichen STS-Literatur zum Thema Einsatz von Informations- und Kommunikationstechnologien (IKT) in der Wissenschaft füllt.

Überraschenderweise war bislang auch die sozialwissenschaftliche Tech- 
nikfolgenabschätzung an der umfassenden Analyse der Folgen des IKT-Einsatzes in der Wissenschaft wenig beteiligt. Zwar gibt es eine Reihe von Studien zu einzelnen Aspekten, etwa zur elektronischen Lehre (z. B. OTA 1988), zu Hochleistungsrechnern und Netzwerken (OTA 1991), zu elektronischen Büchern (Riehm et al. 1992; Böhle et al. 1997) und zu urheberrechtlichen Aspekten (Banse/Langenbach 1999; OTA 1986). In all den zitierten Projekten ging es jedoch nicht primär um die Wissenschaft im Speziellen.

Ein interdisziplinär angelegtes, mehrjähriges Forschungsprojekt zum Thema "Cyber-Wissenschaft - Der Einfluss der Informations- und Kommunikationstechnologien auf die Wissenschaft", durchgeführt am Institut für Technikfolgen-Abschätzung (ITA) der Österreichischen Akademie der Wissenschaften, hat sich der Aufgabe gestellt, beide Lücken zu füllen (Nentwich $2003)^{2}$. Da es sich bei diesem Forschungsthema freilich nicht um rein österreichische Fragestellungen handelt, erfolgte die Durchführung teilweise in internationaler Kooperation (mit dem Max-Planck-Institut für Gesellschaftsforschung in Köln), durch empirische Erhebungen im weltweiten Internet sowie durch Interviews auch mit internationalen ForscherInnen. Die insgesamt 50 Interviews wurden auf Basis eines halbstrukturierten Leitfadens durchgeführt. Das Gesamtprojekt und damit auch der vorliegende Artikel beruhen weiters auf einer breiten Literaturrecherche sowie auf Selbst- und teilnehmender Beobachtung.

Im Sinne des umsetzungsorientierten, auf die Beratung der Politik gerichteten Vorgehens der Technikfolgenabschätzung können die allgemeinen Aussagen hier auch in Hinblick auf Handlungsbedarf in Österreich spezifiziert werden (siehe unten 4). Zunächst wird jedoch kurz auf den Status Quo der Internet-basierten Kommunikationstechnologien in den Wissenschaften eingegangen (2), gefolgt von einer Diskussion der Folgen der Techniknutzung (3).

\section{Internet-basierte Kommunikationstechnologien in den Wissenschaften}

Die Entwicklung des Internet ist eng mit dem kreativen Potential der Wissenschaften verknüpft: So wurden wesentliche Elemente des heutigen Internet (z. B. E-Mail, WWW) von WissenschafterInnen für Wissenschafterlnnen entwickelt. Umgekehrt sind auch manche Entwicklungen in der Wissenschaft Ende des 20., Anfang des 21. Jahrhunderts nur verstehbar, wenn man die Auswirkungen der neuen IKT mitberücksichtigt (zu den Folgen unten 3.). 
Elektronische Post hat heute in praktisch allen wissenschaftlichen Disziplinen andere Formen der schriftlichen Kommunikation (Fax, Brief) weitgehend überlagert. Auf E-Mail basierende elektronische Foren (also vor allem discussion lists, weniger verbreitet hingegen newsgroups, blogs und chat) variieren zwar in ihrer Bedeutung, doch kein Feld kommt ganz ohne diese neuartigen Kanäle des Austauschs von Informationen aller Art und der Diskussion von fachspezifischen Fragen aus. Elektronische Publikationsformen haben ebenfalls weite Verbreitung gefunden. Hier gibt es zwar große Unterschiede zwischen den einzelnen Fachbereichen, aber ein eindeutiger Trend ist überall auszumachen. Insbesondere elektronische Arbeitspapiere und die dazugehörigen so genannten E-Pre-Print-Archive haben in manchen Bereichen die traditionellen, papiergebundenen Formen weitgehend ersetzt (Nentwich 2003, 139 ff.). Das elektronische Zeitschriftenangebot und damit der direkte Zugang zu den aktuellen wissenschaftlichen Veröffentlichungen ist bereits unübersehbar, da die meisten großen Zeitschriftenverlage mittlerweile ihr gesamtes Angebot nicht nur drucken, sondern auch online zur Verfügung stellen. Dazu kommen noch mannigfache, meist von wissenschaftlichen Vereinigungen getragene Initiativen zur Herausgabe von reinen „EJournalen", d. h. solchen, die überhaupt nur online erscheinen. Dementsprechend wurden weltweit hunderte generelle und fachspezifische digitale Bibliotheken aufgebaut, die die elektronisch verfügbaren Informationen, Dokumente und Veröffentlichungen strukturiert aufbereiten und nutzbar machen (z. B. Arms 2000). Weiters stellen fachspezifische Datenbanken, die auch auf Distanz über das Internet abfragbar sind und Primär- und Sekundärdaten zugänglich machen, in den meisten Feldern einen wesentlichen Bestandteil des Forschungsinstrumentariums dar. Wissenschaftliche Veröffentlichungen verwandeln sich auch in ihrer Form. Multimediale Elemente, wie z. B. kurze Video- oder Audiosequenzen oder Hypertextstrukturen, werden teilweise schon intensiv genutzt und eröffnen völlig neue Darstellungsformen.

In einigen Bereichen erfüllen verschiedene Formen des "verteilten Rechnens", d. h. der dezentralen Zurverfügungstellung von Computerleistung und deren zentrale Koordinierung und Auswertung, wesentliche Funktionen in der Forschung (z. B. Foster/Kesselman 1999). Auch die Zusammenarbeit auf Distanz von Forschenden, die zwar gemeinsam, aber nicht an einem physischen Ort vereint an kleineren und größeren Projekten arbeiten, wurde durch das Internet auf eine völlig neue Basis gestellt. Während EMail-basierte Dienste wie erwähnt zum Standardrepertoire gehören, sind fortgeschrittenere Anwendungen noch weniger verbreitet, haben aber vermutlich ein großes Potential. So beginnen sich langsam Groupware-Appli- 
kationen auch in der Wissenschaft durchzusetzen, um Zusammenarbeit auf Distanz zu erleichtern. Internet-basierte elektronische Konferenzen mit synchroner Audio- und Videoübertragung werden zwar kaum je Zusammenkünfte an einem Ort zwischen WissenschafterInnen, sei es zum persönlichen Gespräch, sei es auf Konferenzen oder Arbeitstreffen, ersetzen. Es steht jedoch zu erwarten, dass Web-Konferenzen bald zum wissenschaftlichen Arbeitsalltag gehören werden. Vereinzelt gibt es bereits "virtuelle Forschungsinstitute", also dauerhafte organisatorische Zusammenschlüsse von Forschenden, die physisch über den Erdball verteilt sein können und zu einem hohen Grad nur über elektronische Medien in Austausch treten. Ebenso sind sog. "collaboratories" (Finholt 2001) bereits weit verbreitet, die interessierten WissenschafterInnen weltweit Zugang zu fachspezifischen Ressourcen bieten. Dies kann sogar so weit gehen, dass Laborgeräte und Messinstrumente auf Entfernung bedient werden können (Olson et al. 2006).

Generell lässt sich sagen, dass die Diffusion der Informations- und Kommunikationstechnologien in den Wissenschaften schon weit fortgeschritten ist (Nentwich 2003, Kap. 3). Gleichzeitig sind zum Teil große Unterschiede zwischen den einzelnen Fächern zu beobachten, wobei jedoch nicht unbedingt die naturwissenschaftlichen in jeder Hinsicht die sozial- oder geisteswissenschaftlichen Disziplinen in der Internetnutzung übertreffen (ibid.). So ist heute Papyrusforschung ohne weltweit vernetzte Datenbanken undenkbar, hat die nordamerikanische Geschichtswissenschaft eine Vorreiterrolle bei der Nutzung von Hypermedia-Publikationen und sind etwa einzelne medizinische Spezialgebiete in Hinblick auf die Internetnutzung unterdurchschnittlich innovativ.

\section{Folgen der Technologienutzung}

Dass die Nutzung des Internets den Arbeitsalltag der Forschenden über die letzten eineinhalb Jahrzehnte inkrementell und - so meine These - nachhaltig verändert, erscheint für alle in der Wissenschaft Tätigen auf den ersten Blick plausibel. Doch was bedeutet dieser Wandel konkret? Wie weitgehend sind die Folgen? Könnte es sein, dass die zunächst vor allem quantitativen Veränderungen (z. B. höhere Kommunikationsgeschwindigkeiten) auch eine neue Qualität haben? Werden vielleicht sogar die Inhalte der Forschung selbst berührt, werden wir andere Fragen stellen und andere Antworten geben?

Hier kann nur ein grober Überblick über die wichtigsten handlungsrele- 
vanten Folgen der Internetnutzung in der Wissenschaft gegeben werden, wie sie sich aus der systematischen Analyse der ITA-Studie (Nentwich 2003) ergeben. Ich diskutiere zunächst zwei genuin soziologische Aspekte, nämlich die Auswirkungen auf die Rollenverteilung im wissenschaftlichen Betrieb (3.1) und die Wirkungen des verbesserten Zugangs zu Internetressourcen auf das wissenschaftliche Hierarchiesystem (3.2). Daran anschließend folgen drei wichtige, mit den Veränderungen des wissenschaftlichen Publikationswesens einhergehende Themenfelder: die Notwendigkeit der Archivierung der zunehmend digitalen Bestände (3.3), die Bedeutung des Urheberrechts in diesem Zusammenhang (3.4) und die Qualitätssicherung in jenem Teil des Internet, welches die Wissenschaften zu ihrer internen Kommunikation benutzen (3.5).

\subsection{Rollen- und Funktionswandel}

Der zunehmende Einsatz von IKT wirkt sich auf die Rollenverteilung in der Wissenschaft sowie auf die Anforderungen aus, die an einzelne Personengruppen gestellt werden. Man kann beobachten, dass die Forschenden eine Reihe von Aufgaben übernommen haben, die früher vom Sekretariatspersonal, von Bibliothekaren oder von Verlagen erledigt wurden. Dies betrifft vor allem den Schriftverkehr, die Informationsbeschaffung und das Formatieren von Publikationen (Grötschel/Lügger 1996; Mueller 2000). In Zukunft steht zu erwarten, dass noch weitere Tätigkeiten hinzukommen werden. Das bedeutet, dass die Rolle des/r Wissenschafters/in in Veränderung begriffen ist. Dazu kommt noch, dass auch deren zweites Tätigkeitsfeld, nämlich die Lehre, ebenfalls neu definiert wird. Nicht nur müssen neue Fertigkeiten erlernt werden, um die Erwartungen der Studierenden zu erfüllen, sondern möglicherweise wird der Schwerpunkt von traditionellen Unterrichtsformen (insbesondere Vorlesungen) in Richtung "Tutoring", also persönlicher Betreuung, Anleitung und Diskussion in Kleingruppen verschoben, während die Grundkurse in elektronischer Form wieder verwendet werden (Massy/ Zemsky 1995). Auch die Anforderungen an akademische Bibliothekare wandeln sich grundlegend. Die Cyber-Bibliothekare („Cybrarians“ - Okerson 1997a; Johnston 1998) wandeln sich zu Computerexperten und Aufbereitern von Informationen ("Information Brokers") im digitalen Wissensraum, den sie federführend mitstrukturieren. Auch werden sie vermutlich grundlegende Aufgaben im akademischen Veröffentlichungswesen übernehmen und sogar unterrichten (nämlich Online-Informationssuche).

Überhaupt ist der Bereich der Veröffentlichungen aufgrund der neuen 
Möglichkeiten des elektronischen Publizierens und der finanziellen Krise des akademischen Marktes (z. B. Okerson 1997b) in grundlegendem Wandel begriffen. Die traditionelle Funktion der wissenschaftlichen Verlage, insbesondere der kommerziellen, könnte durch die Open-Access-Bewegung (z. B. Bullinger/al. 2003) zurückgedrängt zu werden. Deren Tätigkeitsfeld wird sich nach einer These auf aufwendige Nischenprodukte reduzieren (Nentwich 2001). Wissenschaftliche Gesellschaften, Universitäten, Bibliotheken und einzelne Wissenschafterlnnen übernehmen teilweise Verlagsaufgaben und verringern damit deren Einfluss. Die Rolle der (Zeitschriften)Agenten wird zunehmend von den Verlagen selbst oder den Bibliotheken wahrgenommen.

\subsection{Zum Verhältnis Zentrum-Peripherie: \\ Zugangsfragen und soziales Kapital}

Mit der wachsenden Bedeutung des Internet für die Wissenschaft (Informationen, Datenbanken, Publikationen, Rechenressourcen etc.; siehe oben 1), wird der möglichst ungehinderte Zugang zum Netz immer mehr zu einer wesentlichen Voraussetzung für das erfolgreiche Betreiben von Wissenschaft (wobei selbstverständlich die gravierenden Unterschiede zwischen den verschiedenen Disziplinen hinsichtlich des Informationsbedarfs etc. in Rechnung gestellt werden müssen). Während der rein physische Zugang (d. h. ein Internetanschluss) selbst in den Labors und Forschungsinstituten der sog. Entwicklungsländer heute zumeist vorhanden ist, stellt sich die Zugangsproblematik auf der nächsten Ebene. Zwar sind viele (auch hoch qualitative) Informationsangebote frei zugänglich, doch können viele Datenbanken, Publikationen und sonstige Ressourcen nur gegen Entrichtung teils hoher Gebühren benutzt werden. Neben den Kosten für die Aufrechterhaltung eines leistungsfähigen technischen Zugangs zum Netz fallen diese Lizenz- und Nutzungsgebühren stark ins Gewicht. Gerade die steigenden Lizenzgebühren für Zeitschriftenabonnements und Index-Datenbanken stellen oft eine große Zugangshürde dar (Walker 1998). Diese digitale Spaltung ("digital divide") auf der zweiten Ebene ist im Übrigen nicht nur im Verhältnis zwischen den Forschungszentren der sog. Ersten und Dritten Welt zu beobachten, sondern auch innerhalb der Ersten: Es gibt etwa auch in Westeuropa "periphere" Institute, d. h. solche, die unterausgestattet sind und dementsprechend nicht aktiv an der Spitze der Forschung partizipieren können.

Es wurde diskutiert, ob der Einsatz von IKT die Perspektiven für periphere Institute verbessern könnte (z. B. Matzat 2001; Finholt/Olson 1997). Einer- 
seits ist festzuhalten, dass die Kosten des Zugangs zu wissenschaftlichen Informationen durch Anschluss an das Internet geringer sind als jene für den Aufbau einer entsprechenden lokalen Infrastruktur. Ob steigende Lizenzgebühren diese Vorteile wieder aufheben werden, ist noch nicht endgültig zu beantworten. Dazu kommt noch, dass in vielen Fächern zumindest vorläufig bei weitem noch nicht alles Relevante digital und online verfügbar ist, was den Gesamteffekt derzeit noch einschränkt.

Zugang ist freilich nicht nur auf die formelle wissenschaftliche Kommunikation bezogen, sondern spielt auch im informellen Bereich eine Rolle. Internet-basierte Kommunikation ermöglicht in bislang unmöglichem Ausmaß Networking innerhalb wissenschaftlicher Gemeinschaften. Ebenso kann der Zugang zu bisher oftmals schwer zugänglichen, weil hierarchisch kontrollierten Informationsressourcen (etwa zu Calls for Papers) ebenso wie besonderes technisches Know-How (gepaart mit entsprechendem Engagement) zum Aufbau von sozialem Kapital führen, das in der Offline-Welt kaum akkumulierbar wäre.

Es stellt sich allerdings die Frage, welchen Stellenwert der Zugang zu Informationen insgesamt überhaupt hat. Offensichtlich haben der direkte, persönliche Kontakt mit den zentralen Akteuren, die Gespräche in den Korridoren und Teeküchen einen großen Anteil an der Positionierung einer Forschungseinrichtung (Walsh/Roselle 1999; Merz 1998). Partizipation in diesem informellen Prozess ist essentiell, und elektronische Medien könnten ungeeignet sein, diese in gleicher Weise zu unterstützen. Zwar zeigen empirische Untersuchungen, dass periphere Einrichtungen und Forschende verstärkt am Kommunikationsprozess teilnehmen (Matzat 1999), dies aber letztlich zu keiner Veränderung der Statushierarchie führt (Fröhlich 1996). Der Hauptgrund dafür ist, dass die Top-Institutionen parallel ebenso vom IKT-Einsatz profitieren, sodass es lediglich zu einer Niveauverschiebung kommen wird, nicht aber zu einer Verringerung oder gar Aufhebung der Abstände.

\subsection{Digitale Reorganisation der Archivierung}

Im Unterschied zur wohl etablierten Archivierung von Printpublikationen steckt die digitale Archivierung noch in den Kinderschuhen. Sowohl in technischer als auch in organisatorischer Hinsicht handelt es sich um kein einfaches Problem, da eine sehr langlebige, nachhaltige und sichere Lösung gefunden werden muss. Dennoch scheinen die technischen Schwierigkeiten (Vorsorge gegen das "Verschwinden" von Dateien, die Erhaltung der ent- 
sprechenden Soft- und eventuell auch der Hardware, um digitale Daten auch auf späteren Maschinen lesbar zu halten, die Vergänglichkeit der Speichermedien usw.) prinzipiell lösbar. Vorgeschlagen und teilweise implementiert wurden Spiegelserver, Sicherungskopien, zusätzliche Papierarchive, die Emulation von alter Software auf neueren Maschinen usw. (Rauber/Aschenbrenner 2001). Davon abgesehen stellen sich vor allem zwei Fragen: (1) Was soll überhaupt archiviert werden? (2) Wie kann das langfristig organisatorisch sichergestellt werden?

ad (1) Es scheint unmöglich und auch wenig sinnvoll, prinzipiell alles, was je im Internet veröffentlicht wurde, auch zu archivieren (Risak 2000). Es müssen somit Auswahlentscheidungen getroffen werden. Während bei den formellen wissenschaftlichen Publikationen kaum Zweifel aufkommen werden, gibt es einige weitere Kategorien, wo konkrete Entscheidungen anstehen: akademische Software samt den dazugehörigen Datensammlungen; Vorveröffentlichungen; Konferenzbeiträge; Arbeitspapiere; Beiträge zu akademischen E-Mail-Diskussionslisten; Homepages von Forschungseinrichtungen; frühere Fassungen von veröffentlichten Artikeln; akademische Linksammlungen; und Quellenarchive. Eine Richtschnur könnte es sein, jedenfalls all das dauerhaft zu erhalten, was an irgendeiner Stelle Eingang in den wissenschaftlichen Diskurs gefunden hat, also z. B. zumindest einmal zitiert wurde. Die Art der Realisierung dessen ist noch offen. Es kommen sowohl unspezifische Lösungen wie die periodische Gesamtarchivierung des gesamten Internetgehalts, als auch strukturiert gesammelte Online-Archive der großen wissenschaftlichen Bibliotheken in Frage.

ad (2) Weiters müssen Verantwortlichkeiten festgelegt werden, um auch organisatorisch sicherzustellen, dass die wissenschaftliche Gemeinschaft nicht Teile ihres verschriftlichten Erbes verliert. Während jene Dateien, die oft nachgefragt werden, vermutlich ohne organisatorische Vorkehrungen durch die NutzerInnen und Anbietenden archiviert und zugänglich gehalten werden, ist dies für selten genutzte Ressourcen nicht zu erwarten (Dementi 1998). Insbesondere haben die Verlage selbst kein langfristiges Eigeninteresse an der dauerhaften Archivierung „überholter" oder vergriffener Bücher und Zeitschriften. Dazu kommt noch, dass Verlage auch Marktbereinigungen zum Opfer fallen können. Demgegenüber gibt es weitgehend noch keine Verpflichtung zur Ablieferung von Pflichtexemplaren an besondere Bibliotheken. Andererseits sehen dies die wissenschaftlichen Bibliotheken als ihre Kernaufgabe (Atkinson 1996), ohne jedoch im digitalen Bereich über ausreichende Rechte zu verfügen. Das bei digitalen Publikationen übliche restriktive Lizenzsystem kann nämlich bedeuten, dass nach Ablauf der Lizenz das Produkt nicht mehr zugänglich gemacht werden darf. Jedenfalls 
besteht hier großer Koordinationsbedarf, damit es angesichts des ohnehin schon riesigen Aufwands nicht zu unnötigen Verdoppelungen kommt.

\subsection{Reformbedarf im (Urheber-)Recht}

Wissenschaft, insbesondere wissenschaftliches Publizieren findet nicht im rechtsfreien Raum statt. Die neuen Möglichkeiten elektronischer Veröffentlichungen trafen auf eine Rechtslage, die noch durch die nicht-digitale, offline-Welt der Druckwerke geprägt war. Mittlerweile sind mannigfache legistische Aktivitäten im Gange, um das Recht des intellektuellen Eigentums an die neuen Gegebenheiten anzupassen. Da hier auch gewichtige kommerzielle Interessen im Spiel sind (vor allem auch außerhalb der Wissenschaft Stichwort Raubkopien), sind die Gesetzgeber auf der ganzen Welt geneigt, strenge Bestimmungen zu erlassen, die jedoch oftmals nicht im Interesse der Wissenschaft liegen. Diese benötigt nämlich möglichst freien Zugang zu den digitalen Wissensbeständen. Diesbezügliche Initiativen (Open Archives, Open Access, Public Library of Science, ELSSS, BiomedCentral etc.) aus der Wissenschaft, teilweise unter Beteiligung von Nobelpreisträgerlnnen und den großen wissenschaftlichen Organisationen, versuchen hier ein Gegengewicht zu schaffen.

Weiters stellte sich im Zuge unserer empirischen Erhebungen heraus, dass Rechtssicherheit für die meisten eine essentielle Voraussetzung für den Übergang zu elektronischen Publikationsformen darstellt. Auch wenn das Internet keineswegs als rechtsfreier Raum bezeichnet werden kann, seien hier von den zurzeit noch offenen Punkten einige herausgegriffen (genauer in Nentwich 2003, Kap. 9.2):

(1) Nicht einheitlich geregelt und offensichtlich teilweise unzulässig ist das ausführliche Zitieren von digitalen Quellen, ohne dafür lizenzpflichtig zu werden (Nentwich 2004). Müsste für jedes Zitat bezahlt werden oder auch nur die Genehmigung eingeholt werden, wäre Wissenschaft, wie wir sie bislang kennen, praktisch verunmöglicht. Insbesondere für MultimediaPublikationen, die auch in der Wissenschaft immer häufiger werden, kann es zu Schwierigkeiten kommen, da diese mitunter aus vielen Elementen bestehen, für die nicht einmal das bisher gültige Recht des freien Zitats anwendbar ist.

(2) Die in vielen Fällen sinnvolle Digitalisierung von älteren Beständen (etwa von früheren Jahrgängen wissenschaftlicher Zeitschriften) stößt oft an die engen Grenzen der urheberrechtlichen Zulässigkeit, da es praktisch unmöglich sein kann, die entsprechenden Rechte einzuholen. 
(3) Nicht restlos geklärt bzw. zu klären (weil es sich nicht zuletzt um individuelles Vertragsrecht handelt) ist die Frage der Zulässigkeit der Selbstarchivierung von bereits publizierten Texten durch die Autorlnnen in öffentlichen Webarchiven. Deren Funktion ist es, den Zugang zu publizierten ebenso wie zu (noch) nicht publizierten Forschungsergebnissen unabhängig von Verlagen und Bibliotheken auf Dauer sicherzustellen.

(4) Strenge Urheberrechtsbestimmungen könnten schließlich die wissenschaftliche Kreativität behindern, da wissenschaftliche Publikationen immer öfter in einem interaktiven, iterativ-sequentiellen Prozess entstehen. Hier sind neue Modelle gefragt, die verhindern, dass durch die Zuerkennung von individueller Urheberschaft an Bestandteilen des Ganzen, das Aufeinanderaufbauen und Fortentwickeln behindert wird.

(5) Ein besonders wichtiges Problem in diesem Zusammenhang ist die mangelnde Transparenz, da die AnwenderInnen (in der Forschung) in der Regel keine spezielle juristische Bildung haben. Insbesondere aufgrund der nur teilweisen internationalen Harmonisierung ist die Rechtslage jedoch komplex; aufklärerische Projekte wie Knowledge Base Copyright Law (KB:Law|C) sollen hier Abhilfe schaffen (Nentwich 2006).

\subsection{Qualitätssicherung im Internet als Herausforderung}

Die Kontrolle der Qualität der veröffentlichten Forschungsresultate nimmt eine zentrale Stellung in der Wissenschaft ein. Die scheinbar unbegrenzten Möglichkeiten zur Eigenpublikation im Internet lassen diese Aufgabe noch bedeutender werden. Während es im Bereich der formellen Veröffentlichungen (insbesondere E-Zeitschriften, E-Bücher) problemlos möglich ist, die in der Papierwelt etablierten Verfahren des Peer Review zu übertragen, werden für neuartige digitale Publikationsformen neue Qualitätssicherungsmaßnahmen gesetzt, die zum Teil neben die herkömmlichen Formen treten können (Nentwich 2005). Unter dem Stichwort "Open Peer Review“ werden zumeist nicht-anonyme Prozesse des öffentlichen Kommentierens über eingereichte Manuskripte, die zu diesem Zweck online gestellt wurden, verstanden. Dabei wird den HerausgeberInnen der Zeitschrift jedoch nach wie vor die Letztentscheidung auf Basis der eingetroffenen Kommentare überlassen (Sumner/Shum 1997). Daneben gibt es die Qualitätskontrolle nach der Veröffentlichung in Form von öffentlichen Kommentaren und Benotungen, die den LeserInnen der ursprünglich nicht referierten Arbeiten eine Einschätzung der Qualität erlaubt (LaPorte et al. 1995). Im Unterschied zum traditionellen Zirkulieren und Kommentieren grauer Veröffentlichungen be- 
steht in der Online-Welt das Potenzial zu etwas Neuem: Der Diskurs wird öffentlich geführt und dokumentiert, was bislang nur sehr eingeschränkt möglich war, und damit potenziell gestärkt. Aus quasi-einseitiger Publikation wird im digitalen Raum multilaterale Kommunikation (LaPorte et al. 1995).

Im Prinzip ähnlich, jedoch formaler, funktionieren Qualitätssicherungssysteme, die die Nutzung von Veröffentlichungen beobachten (z. B. Zahl der Zitierungen bzw. Links zu einem Artikel oder Anzahl derjenigen, die ihn aufgerufen haben). All diese Daten zu jedem Artikel können die Grundlage für selektive Datenbanken bilden, deren Filter die Lesenden so einstellen können, dass beispielsweise nur doppelt-blind referierte Artikel oder nur solche, die eine bestimmte Nutzungsfrequenz oder Anzahl von Zitaten erreicht haben, angezeigt werden (Nentwich 1999).

Weiters sind einfache Formen von Plagiaten in einem voll digitalisierten Veröffentlichungswesen automatisiert auffindbar (Harnad 1998). Damit würde der oft geäußerten Befürchtung, dass das Internet Plagiate durch die Erleichterung der Zugänglichkeit und des Kopiervorgangs unterstützt, in Zukunft die Grundlage entzogen. Ironischerweise erlaubt somit die Digitalisierung der wissenschaftlichen Veröffentlichungen unter bestimmten Rahmenbedingungen sogar mehr, nicht weniger Qualitätskontrolle (Nentwich 2005).

\subsection{Zwischenresümee}

Neben den oben diskutierten Konsequenzen der zunehmenden Internetnutzung in den Wissenschaften gibt es freilich noch weitere, die hier aus Platzgründen ausgeklammert werden müssen, etwa in Bezug auf die Kooperationsbeziehungen zwischen WissenschafterInnen, die universitäre Infrastruktur, das Wissensmanagement innerhalb von Forschungsinstitutionen oder generell die Zukunft von papiergebundenen Publikationen in der Wissenschaft (siehe Nentwich 2003). Dies alles trägt zu einem eindeutigen Gesamtbefund bei: Das wissenschaftliche System ist in einem tief greifenden Wandel begriffen. Der Einsatz von Informations- und Kommunikationstechnologien in der Wissenschaft kann nicht nur zu quantitativen Veränderungen (etwa Effizienzsteigerungen oder die Vergrößerung des Kreises an Kommunikationspartnern) führen, sondern hat auch strukturelle Auswirkungen zur Folge, die das Wissenschaftssystem insgesamt umgestalten. Auch wenn der Technologieeinsatz nicht als der einzige Faktor für diesen Wandel angesehen werden darf (so spielen unter anderem etwa auch Finanzierungsstra- 
tegien oder das generelle Anwachsen des Sektors eine Rolle), so bleibt doch festzuhalten, dass das Internet einen gestaltenden Einfluss auf die Art und Weise, wie Wissenschaft am Anfang des 21. Jahrhunderts betrieben wird, hat.

Im abschließenden Kapitel 4 werde ich im Sinne des Anspruchs von Technikfolgenabschätzung, handlungsorientiertes Wissen zu produzieren, Optionen und Notwendigkeiten für die Politik aufzeigen.

\section{Handlungsoptionen und -notwendigkeiten}

Die Entwicklung hin zur Cyber-Wissenschaft verläuft derzeit noch weitgehend ungesteuert und war bislang nur punktuell Gegenstand politischen Handelns, so etwa im Infrastrukturbereich (Stichwort: Grid-Computing). Abschließend soll daher die Frage nach den wichtigsten Themen für die Politik gestellt werden - verstanden in einem weiten Sinne, d. h. auch die Standespolitik oder die inneruniversitäre Politik einbeziehend. Die wichtigsten Themen, die sich unter anderem aus den oben diskutierten Folgen des Technologieeinsatzes in der Wissenschaft ergeben, können in drei Gruppen eingeteilt werden: (1) das Zurverfügungstellen einer adäquaten Infrastruktur, einschließlich eines sicheren und universellen Zugangs zu dieser; (2) die Gestaltung einer vertrauenswürdigen Umgebung für elektronisches Publizieren; und (3) die Einrichtung von akademischem Informationsmanagement und die dafür notwendige Ausbildung. Wir werden zunächst die Handlungsoptionen und -notwendigkeiten in diesen drei Bereichen darstellen und sie anschließend verschiedenen Akteuren zuordnen.

ad (1) Zunächst ist festzustellen, dass Cyber-Wissenschaft stark von einer leistungsfähigen und sicheren Infrastruktur abhängig ist. Daher gibt es weltweit Bemühungen um breitbandige wissenschaftliche Hochgeschwindigkeitsnetze, die sowohl verteiltes Rechnen auf höchstem Niveau und raschen Austausch von großen Datenmengen als auch gute Echtzeit-Videoverbindungen erlauben (OECD 1998; European Commission 2002). Viele Länder haben erkannt, dass manche wissenschaftliche Fächer ohne Anschluss an diese Netze nicht mehr wettbewerbsfähig sein können (und dass andere Fächer durch eine Art Sogwirkung ebenfalls profitieren könnten). Österreich hat etwa in Hinblick auf das europäische Hochgeschwindigkeitsnetz GÉANT noch Nachholbedarf.

In Hinblick auf den Aufbau der Cyber-Infrastruktur spielt auch die Abgrenzung des öffentlich finanzierten Wissenschaftsnetzes von den kommer- 
ziellen Netzen eine wichtige Rolle, sowohl in Hinsicht auf die Hardware als auch auf die Software (Stichwort: Open Source), um dessen Unabhängigkeit zu garantieren.

Abgesehen von der weltweiten Ungleichverteilung des Zugangs zum Internet ist auch der Zugang zu höherwertigen Diensten in der entwickelten Welt nicht gleich verteilt. Insbesondere der universelle Zugang zu Datenbanken (Tauss 2001; Arzberger et al. 2004; Wouters/Schröder 2003) und zur essentiellen Forschungsliteratur ist keineswegs gesichert, sondern aufgrund gravierender ökonomischer Interessen im Verlagswesen (sehr hohe und weiter steigende Preise für digitale Abonnements) gefährdet. Österreich ist hier keine Ausnahme. Selbst in großen außeruniversitären Forschungseinrichtungen ist der Zugriff auf alle relevanten Fachzeitschriften keineswegs selbstverständlich.

ad (2) Der nicht überall linear verlaufende, aber nichtsdestotrotz zügig fortschreitende Umbau des wissenschaftlichen Publikationswesens von Papier- zu elektronischen Medien gibt Anlass, sich mit dessen Folgen auch unter Steuerungsgesichtspunkten auseinander zu setzen. Aufgrund der überragenden Bedeutung des Publikationswesens für die Wissenschaften ist der Umstieg vom bewährten Papiersystem zum Internet vorsichtig umzusetzen. Das neue System muss sowohl stabil als auch vertrauenswürdig sein, um angenommen zu werden. Hier sind sowohl technische als auch rechtliche, ökonomische und organisatorische Aspekte zu beachten. Insbesondere das Urheberrecht und sonstige einschlägige Bestimmungen müssten den Erfordernissen des wissenschaftlichen Arbeitens im Internet angepasst werden. Hier ist notabene internationale Akkordierung (EU, OECD) notwendig, um das notwendige weltweit sichere Umfeld zu schaffen.

Um Vertrauen zu schaffen, müsste im elektronischen Bereich zumindest ein gleiches Maß an Qualitätskontrolle wie im traditionellen System sichergestellt werden (Atkinson 1996). Die bereits oftmals erfolgte Übertragung des Peer-Review-Systems auf die E-Journale kann als erster notwendiger Schritt betrachtet werden. Es liegt aber auch nahe, die neuen Möglichkeiten des öffentlichen Ex-ante- und Ex-post-Kommentierens sowie von RatingSystemen etc. (oben 3.5) auszuschöpfen, um Lücken zu schließen (etwa in Hinblick auf Arbeitspapiere).

Da elektronische Publikationen neben den Vorteilen für die Wissensrepräsentation und den Zugriff auf Informationen ein großes Einsparungspotential bergen, ist die Bewertung dieser wie Papierpublikationen und die Schaffung von Anreizen für die neuen Formen ein Thema.

Erhebliche Anstrengungen sind schließlich im Bereich der Sicherstellung der langfristigen Archivierung vonnöten. Insgesamt könnte eine nachhaltige 
Strategie der Dekommodifizierung, d. h. der vorsichtigen Herauslösung des wissenschaftlichen aus dem kommerziellen Publikationssektor, zur Stabilisierung und Sicherstellung dieses wichtigen Eckpfeilers der Wissenschaft führen (Tehranian 1996).

ad (3) Informationsmanagement war immer ein wesentlicher Bestandteil wissenschaftlicher Tätigkeit. Jetzt, da Informationen zunehmend digital abgespeichert werden, stellen sich neue Herausforderungen, sowohl auf individueller als auch auf kollektiver Ebene. Während die Aufgaben prinzipiell gleich bleiben, ändern sich die Methoden, Arbeitsweisen und Optionen. Informations- bzw. Contentmanagementsysteme wurden bislang in der Wissenschaft noch kaum genutzt (Fröhlich 1996). Angesichts der primären Unübersichtlichkeit des Informationsangebots im Internet gibt es jedoch großes Potential, was nicht zuletzt in der Semantic-Web-Initiative des WWW-Konsortiums zum Ausdruck kommt (Berners-Lee 1998). Das betrifft sowohl die wissenschaftlichen Bibliotheken, die sich als Informationsmittler im digitalen Raum begreifen, als auch Aktivitäten auf der Ebene wissenschaftlicher Disziplinen, die über bloße Linksammlungen in Richtung thematischer Datenbanken hinausgehen. Schließlich muss betont werden, dass der Umgang mit digitalen Informationen auch Schulung und Beschäftigung mit dem Thema der Wissensstrukturierung auf der Metaebene erfordert (Grötschel/ Lügger 1996). Auf diese Weise können nicht nur Ineffizienzen vermieden, sondern auch die dynamische Entwicklung des Informationsraums entsprechend den Bedürfnissen der Wissenschaft mitgestaltet werden. Die Möglichkeiten reichen hier von einschlägigen Pflichtlehrveranstaltungen bei der Universitätsausbildung bis zur Personalaufstockung in den unterstützenden Einheiten (Grötschel/Lügger 1996; Fröhlich 1992).

Nach diesem kursorischen Überblick über Handlungsoptionen muss die Frage gestellt werden, ob und in welcher Weise es geboten ist, eine "Cyber"Wissenschaftspolitik ins Auge zu fassen. Da es sich um ein dynamisches, in Entwicklung befindliches Gebiet handelt, ist von statischer Regulierung abzuraten, nicht zuletzt um den schöpferischen Freiraum nicht einzuschränken (z. B. Dewar 1998). Vielmehr sollten Anreize geschaffen werden, diese Entwicklungen dort zu unterstützen, wo Verbesserungen des Status Quo zu erwarten sind. Dies betrifft zum einen Verbesserungen in der Zugänglichkeit der wissenschaftlichen Literatur und deren Qualität und Aktualität, zum anderen Ausbildungsmaßnahmen, die zu einem informierten, professionellen Umgang mit den neuen Medien führen sollten. Teilweise kann dieses Ziel freilich nur durch finanzielle Unterstützung erreicht werden, etwa im Bereich der Infrastruktur. Andererseits sollten Maßnahmen gesetzt werden, um mögliche negative Auswirkungen zu vermeiden. Hier ist insbesondere an 
die Qualitätssicherung, die Vermeidung von digitaler Spaltung und die Archivierung zu denken. Unterschieden nach den verschiedenen Akteuren einer umfassend verstandenen Wissenschaftspolitik kommen wir zu folgenden Schlussfolgerungen:

(i) Aufgrund der Größe der Aufgabe, der Einordnung derselben als Infrastrukturmaßnahme und der Tatsache, dass es hier weitgehend um grenzüberschreitende Projekte geht, liegt es nahe, die öffentlichen Akteure (Wissenschaftsverwaltungen, nationale und internationale Gesetzgeber) für die Finanzierung einer stabilen und leistungsfähigen IKT-Infrastruktur, sowohl auf Netzwerk- als auch auf Anwenderseite, verantwortlich zu erklären. Weiters könnten diese die weltweiten Aktivitäten in Hinblick auf die Interoperabilität der Infrastruktur und der offenen Gestaltung der Dateiformate unterstützen. Innerhalb der Wissenschaften sollte allgemeiner Zugang zur CyberInfrastruktur sichergestellt werden. Es wäre überlegenswert, dies eventuell sogar auf Basis von spezifischen Subventionen für Datenbanken zu unterstützen. Schließlich sind legistische Maßnahmen im Bereich des Urheberrechts notwendig, um die Wissenschaft so weit wie möglich von der Ausrichtung des Rechts auf kommerzielle Zwecke, die außerhalb der Wissenschaft bestimmend sind, abzuschirmen.

(ii) Das Ziel der Fachgesellschaften auf nationaler und internationaler Ebene sollte weiterhin in der Aufrechterhaltung der Qualitätskontrolle liegen, da davon die Reputation der neuen Medien entscheidend mit beeinflusst wird. Angesichts der Herausforderungen durch das digitale Publizieren wird vorgeschlagen, disziplinenspezifische Qualitätspolitiken für das elektronische Publizieren zu formulieren und zu implementieren. Bislang sind nur wenige derartige Politiken formuliert und implementiert worden, in Österreich fehlen sie soweit ersichtlich noch weitgehend. Weiters könnten die Gesellschaften durch die Herausgabe von referierten Open-Access-Journalen und durch eine aktive Digitalisierungs- und Archivierungspolitik zum Aufbau einer leistbaren und leistungsfähigen Infrastruktur beitragen. Hier gibt es bereits vereinzelte Beispiele (auch in Österreich), es besteht aber noch großes Potential.

(iii) Die wichtigsten Aufgaben von Universitäten und Forschungseinrichtungen im Zusammenhang mit der Entwicklung zur Cyber-Wissenschaft liegen im Bereich der Unterstützung der Einführung von Informationsmanagementwerkzeugen, der Digitalisierung und zumindest mittelfristigen Archivierung ihres eigenen digitalen Angebots, im Bereich der Schulung der Wissenschafter sowie bei der Einrichtung von leistungsfähigen Einheiten zur Unterstützung der Anwender der neuen Medien. Darüber hinaus könnten die Universitäten und sonstigen Träger von Forschung als Arbeitgeber auch 
eine wesentliche Rolle im Bereich der Honorierung von E-Publikationen bei Bewerbungen und Bestellungen spielen, insbesondere bei der Anerkennung von innovativen Formen. Freilich ist Reputation per se nicht verordenbar, aber solange massive Eingangshürden bestehen (wie etwa der kaum zu rechtfertigende Ausschluss von qualitativen E-Journals aus den einschlägigen Zitationsindices), kann Reputation gar nicht erst entstehen.

(iv) Die Forschungsbibliotheken schließlich werden auch im digitalen Raum eine wesentliche Funktion ausüben, insbesondere im Bereich der Koordinierung und Durchführung der weltweiten Archivierung der Forschungsliteratur, im Bereich der Digitalisierung älterer Bestände sowie generell beim Aufbau einer stabilen und vertrauenswürdigen Umgebung für akademische Publikationen.

Auch wenn sich einige dieser Empfehlungen auf die internationale Ebene beziehen, besteht primär Handlungsbedarf auf nationaler und somit auch österreichischer Ebene. Bislang ist die Entwicklung zur Cyber-Wissenschaft abgesehen von Unterstützungsleistungen im Bereich der Hardware-Infrastruktur durch die öffentlichen Hände (sowie teilweise im Bereich des digitalen Lehrangebots durch die Universitäten) weitgehend noch nicht als handlungsrelevant erkannt worden. Um Österreichs Forschung wettbewerbsfähig zu erhalten, sollten in diesem Bereich verstärkt Aktivitäten gesetzt werden. Das bedeutet nicht nur finanzielle Unterstützung. So wichtig diese selbstverständlich ist, sind daneben auch rechtliche Rahmenbedingungen zu adaptieren und wichtige organisatorische Leistungen zu erbringen.

\section{Anmerkungen}

1 Ich danke den beiden anonymen GutachterInnen, die mir wertvolle Hinweise zur Verbesserung dieses Manuskripts sowie Literaturtipps gegeben haben.

2 Das Projekt wurde teils vom ITA eigenfinanziert, teils vom österreichischen Wissenschaftsfonds (FWF-Projekt P 14042-INF) gefördert. Über die Projekt-Homepage können weitere Informationen zum Projekt gefunden werden, insb. eine umfassende Linksammlung sowie der Projektbericht und alle Publikationen im Rahmen des Projekts: http://www.oeaw.ac.at/ita/cyberscience.htm.

Literatur

Arms, W. Y., 2000, Digital libraries, Cambridge, MA/London: MIT Press.

Arzberger, P., Schroeder, P., Beaulieu, A., Bowker, G., Casey, K., Laaksonen, L., Moorman, D., Uhlir, P. und Wouters, P., 2004, An International Framework to Promote Access to Data, Science 303(5665), 1777-1778. 
Atkinson, R., 1996, Library Functions, Scholarly Communication, and the Foundation of the Digital Library: Laying Claim to the Control Zone, The Library Quarterly 66(3), 239-265.

Banse, G. und Langenbach, C. J. (Hg.), 1999, Geistiges Eigentum und Copyright im multimedialen Zeitalter - Positionen, Probleme, Perspektiven - Eine fachübergreifende Bestandsaufnahme; in Reihe: Graue Reihe, Bd. 13, hg. v. Europäische Akademie zur Erforschung von Folgen wissenschaftlich-technischer Entwicklungen, Bad NeuenahrAhrweiler.

Beaulieu, A., 2001, Voxels in the Brain: Neuroscience, Informatics and Changing Notions of Objectivity, Social Studies of Science 31(5), 635-680.

Berners-Lee, T., 1998, Semantic Web Road map, [Aufgerufen am: 06-11-2002] http:// www.w3.org/Designlssues/Semantic.html.

Böhle, K., Riehm, U. und Wingert, B., 1997, Vom allmählichen Verfertigen Elektronischer Bücher - Ein Erfahrungsbericht, Frankfurt/New York: Campus, http://www.itas.fzk.de/ deu/projekt/peb/proda_t.htm,

Bonitz, M., 1979, Wissenschaftliche Forschung und wissenschaftliche Information; in Reihe: Beiträge zur Forschungstechnologie, Berlin: Akademie-Verlag.

Bullinger, H.-J. et al., 2003, Berlin Declaration on Open Access to Knowledge in the Sciences and Humanities (2003-10-22), http://www.zim.mpg.de/openaccess-berlin/.

Dementi, M. A. E., 1998, Access and Archiving as a New Paradigm, Journal of Electronic Publishing 3(3) http://www.press.umich.edu/jep/03-03/dementi.html; auch veröffentlicht in: Paper at the Faxon Institute Colloquium „Electronic Publishing and the Scholarly Communication Process", 7-8 January 1998.

Dewar, J. A., 1998, The information age and the printing press: Looking backward to see ahead; RAND Online Papers, Washington: RAND Corp., http://www.rand.org/ publications/P/P8014/.

European Commission (Information Society Directorate-General), 2002, Research Networking in Europe - Striving for global leadership; Glossy Report, 15.9., Luxembourg: Office for Official Publications of the European Communities, http://www.dante.net/ pubs/ECbrochure.html.

Finholt, T. A., 2001, Collaboratories, in: Cronin, B. (Hg.): Annual Review of Information Science and Technology, (quoted from manuscript), http://intel.si.umich.edu/crew/ Technical\%20reports/Finholt_Collaboratories_10_06_00.pdf.

Finholt, T. A. und Brooks, J. M., 1997, Collaboratory for Research on Electronic Work Analysis of JSTOR: The impact on scholarly practice of access to on-line journal archives, Andrew W. Mellon Foundation Conference "Scholarly Communication and Technology", 1997-04-24/25, Emory Univ., http://www.arl.org/scomm/scat/finholt. html.

Finholt, T. A. und Olson, G. M., 1997, From Laboratories to Collaboratories: A New Organisational Form for Scientific Collaboration, Psychological Science 8(1), 28-36, http://intel.si.umich.edu/crew/Technical\%20reports/Finholt_From_laboratories_to_ collaboratories_07_22_96.pdf. 


\section{Michael Nentwich}

Foster, I. und Kesselman, C. (Hg.), 1999, The Grid: Blueprint for a New Computing Infrastructure, San Francisco: Morgan Kaufmann Publishers.

Fröhlich, G., 1992, Die wissenschaftliche Informationsexplosion - eine Herausforderung für Lehre und Forschung - Zum Symposium, Mangel im Überfluß, Wissenschaftskommunikation im Zeitalter der Informationsexplosion' an der Johannes Kepler Universität Linz, FDZ (3-4), 9-11.

Fröhlich, G., 1993, ,Demokratisierung' der Wissenschaftskommunikation durch Fachinformationssysteme und Computernetze?, in: Institut für Höhere Studien (Hg.): Information und Macht, Wien.

Fröhlich, G., 1996, The (Surplus) Value of Scientific Communication, Review of Information Science 1(II), http://www.inf-wiss.uni-konstanz.de/RIS/1996iss02_01/articles01/ 02.html.

Fry, J., 2003, The cultural shaping of scholarly communication within academic specialisms, Ph. D. thesis, University of Brighton.

Grötschel, M. und Lügger, J., 1996, Neue Produkte für die digitale Bibliothek: die Rolle der Wissenschaften, in: Börsenverein des Deutschen Buchhandels e. V. (Hg.): Die unendliche Bibliothek - Digitale Information in Wissenschaft, Verlag und Bibliothek, Wiesbaden: Harrassowitz, 38-67, http://www.zib.de/groetschel/pubnew/paper/ groetschelluegger1996b_pp.ps.gz; auch veröffentlicht in: Konrad-Zuse-Zentrum für Informationstechnik Berlin (ZIB) Technical Report TR 96-05 (März 1996).

Harnad, S., 1998, On-line journals and financial fire walls, Nature 395(9), 127-128 http:// www.nature.com/cgi-taf/DynaFixer.taf? rqid = /395127A0.frameset.

Harter, S. P., 1996, The Impact of Electronic Journals on Scholarly Communication: A Citation Analysis, The Public-Access Computer Systems Review 7(5), http://info.lib. uh.edu/pr/v7/n5/hart7n5.html.

Hine, C. (Hg.), 2006, New Infrastructure for Knowledge Production: Understanding EScience, Hershey/London/Melbourne/Singapore: Information Science Publishing (Idea Group), http://www.idea-group.com/books/details.asp?id = 5558 .

Joerges, B. und Shinn, T. (Hg.), 2001, Instrumentation between science, state and industry; in Reihe: Sociology of the sciences: a yearbook, Bd. 22, Dordrecht: Kluwer Academic Publishers.

Johnston, C., 1998, Electronic technology and its impact on libraries, Journal of Librarianship and Information Science 30(1), 7-24.

Kilker, J. und Gay, G., 1998, The Social Construction of a Digital Library: A Case Study Examining Implications for Evaluation, Information Technology and LIbraries 17(2), $60-70$.

Kling, R. und McKim, G., 1999, Scholarly Communication and the Continuum of Electronic Publishing, Journal of the American Society for Information Science and Technology 50(9), 890-906, http://xxx.lanl.gov/ftp/cs/papers/9903/9903015.pdf.

LaPorte, R. E., Marler, E., Akazawa, S., Gamboa, C., Shenton, C., Glosser, C., Villasenor, A. und Maclure, M., 1995, The death of biomedical journals, British Medical Journal on- 
Neue Kommunikationstechnologien und Wissenschaft

line 310 (May), 1387-1390, http://www.bmj.com/cgi/content/full/310/6991/1387.

Leydesdorff, L. und Scharnhorst, A., 2003, Measuring the Knowledge Base: A Program of Innovation Studies; Report, im Auftrag von: Bundesministerium für Bildung und Forschung, Amsterdam: Berlin-Brandenburgische Akademie der Wissenschaften, http:// www.sciencepolicystudies.de/Leydesdorff\&Scharnhorst.pdf.

Massy, W. F. und Zemsky, R., 1995, Using Information Technology to Enhance Academic Productivity, Enhancing Academic Productivity, June 1995, Wingspread, http:// www.educause.edu/nlii/keydocs/massy.html.

Matzat, U., 1999, Academic Communication and Internet Discussion Groups: Transfer of Information or Creation of Social Contacts?, ISKO 99 "Globalisierung und Wissensorganisation", 23.-25.9.1999, Hamburg http://www.bonn.iz-soz.de/wiss-org/beitraege/ Abstract.htm.

Matzat, U., 2001, Social networks and cooperation in electronic communities: a theoretical-empirical analysis of academic communication and internet discussion groups; in Reihe: ICS dissertation series, BDBd. 80, HG. hg. v. Interuniversitair Centrum voor Sociaal-Wetenschappelijke Theorievorming en Methodenontwikkeling, Amsterdam.

Merz, M., 1998, ,Nobody Can Force You When You Are Across the Ocean' - Face to Face and E-Mail Exchanges Between Theoretical Phvsicists, in: Smith, C. und Agar, J. (Hg.): Making Space for Science, London: Macmillan, 313-329.

Mueller, M., 2000, The library catalog, the word processor, and the digital archive: Three stages of information technology in humanities scholarship; [Aufgerufen am: 23-10 2000]. URL?

Nentwich, M., 1999, The European Research Papers Archive: Quality Filters in Electronic Publishing, Journal of Electronic Publishing 5(1), http://www.press.umich.edu/jep/ 05-01/nentwich.html.

Nentwich, M., 2001, (Re-)De-commodification in academic knowledge distribution?, Science Studies 14(2), 21-42, http://www.sciencestudies.fi/filestore2/download/230/ Nentwich.pdf.

Nentwich, M., 2003, Cyberscience: Research in the Age of the Internet, Vienna: Austrian Academy of Sciences Press, http://hw.oeaw.ac.at/3188-7.

Nentwich, M., 2004, Wissenschaftliche Praxis im digitalen Zeitalter. Legistische Anmerkungen zum aktuellen österreichischen Urheberrecht, Journal für Rechtspolitik 12(3), $171-176$.

Nentwich, M., 2005, Quality control in academic publishing: challenges in the age of cyberscience, Poiesis \& Praxis. International Journal of Ethics of Science and Technology Assessment 3(3), 181-198, http://dx.doi.org/10.1007/s10202-004-0071-8.

Nentwich, M., 2006, Knowledge Base Law: Ein neuer Weg bei der Wissensvermittlung juristischer Information, in: Schweighofer, E. und Liebwald, D. (Hg.): Tagungsband des 9. Internationalen Rechtsinformatik Symposions IRIS 2006, Stuttgart et al.: Boorberg, im Erscheinen, http://epub.oeaw.ac.at/ita/ita-papers/MN_06_1.pdf.

OECD, 1998, Science, Technology and Industry Outlook 1998 - Chapter 7: The Global 
Michael Nentwich

Research Village: How Information and Communication Technologies Affect the Science System, Paris: Organisation for Economic Co-operation and Development.

Okerson, A. S., 1997a, Introduction to the $6^{\text {th }}$ Edition (1996) of the Directory of Electronic Journals, Newsletters and Academic Discussion Lists; Letzte Aktualisierung: 1997, http://www.people.virginia.edu/ pm9k/libsci/96/intro.html.

Okerson, A. S., 1997b, Midnight in the Garden of Good and Evil? Libraries, Academic Publishing, Copyright, and other Miasmas, http://www.library.yale.edu/ okerson/ okerson-sf.html.

Olson, G., Bos, N. und Zimmermann, A., 2006, Science of Collaboratories, forthcoming.

OTA (U.S. Congress Office of Technology Assessment), 1986, Intellectual Property Rights in an Age of Electronics and Information; Report, Nr. OTA-CIT-302, April, Washington, DC, http://www.wws.princeton.edu/ ota/disk2/1986/8610_n.html.

OTA (U.S. Congress Office of Technology Assessment), 1988, Power On! New Tools for Teaching and Learning; Report, Nr. OTA-SET-379, September, Washington, DC. http:// www.wws.princeton.edu/ ota/disk2/1988/8831_n.html.

OTA (U.S. Congress Office of Technology Assessment), 1991, Seeking Solutions: High Performance Computing for Science; Background Paper, Nr. OTA-BP-TCT-77, March, Washington, DC, http://www.wws.princeton.edu/ ota/disk1/1991/9138_n.html.

Rauber, A. und Aschenbrenner, A., 2001, Part of Our Culture is Born Digital - On Efforts to Preserve it for Future Generations, Trans, Internet-Zeitschrift für Kulturwissenschaften (10), http://www.inst.at/trans/10 Nr/rauber10.htm.

Riehm, U., Böhle, K., Gabel-Becker, I. und Wingert, B., 1992, Elektronisches Publizieren - eine kritische Bestandsaufnahme, Berlin/Heidelberg et al.: Springer.

Risak, V., 2000, Langfristige Wissensspeicherung gestern und heute, computer kommunikativ, 5, 19-23.

Sumner, T. und Shum, S. B., 1997, From Documents to Discourse: Shifting Conceptions of Scholarly Publishing, CHI '98, 1998-04-18/23, Los Angeles, http://kmi.open.ac.uk/tr/ abstracts/kmi-tr-50-abstract.html.

Tauss, J., 2001, Szenario 2010 - Anmerkungen aus der Perspektive der Politik: Wissenschaftspublikationen im digitalen Zeitalter, Wiesbaden: Harrassowitz, 205-218.

Tehranian, M., 1996, The End of University?, The Information Society 12, 441-447.

Walker, T. J., 1998, Free Internet Access to Traditional Journals, American Scientist 86(5), http://www.sigmaxi.org/amsci/articles/98articles/Walker.html.

Walsh, J. P., 1997, Telescience: The Effects of Computer Networks on Scientific Work; consultancy report, mimeo, im Auftrag von: OECD, April 1997: University of Illinois at Chicago.

Walsh, J. P. und Roselle, A., 1999, Computer Networks and the Virtual College, Science Technology Industry Review (OECD) (24), 49-78.

Wouters, P. und Schröder, P. (Hg.), 2003, Promise and practice in data sharing; in Reihe: The Public Domain of Digital Research Data, hg. v. Wouters, P. und Schröder, P., Am- 
Neue Kommunikationstechnologien und Wissenschaft sterdam: NIWI-KNAW.

Zelman, A. G., 2002, Mediated Communication and the Evolving Science System: Mapping the Network Architecture of Knowledge Production, Ph. D. thesis, University of Amsterdam, http://www.rozenbergps.com/files/zelman.pdf. 
